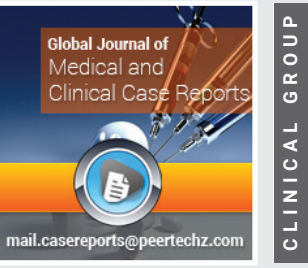

\title{
Adductor spasmodic dysphonia - A detailed case report on \\ assessment and management
}

\author{
Sandhya Koteshwara ${ }^{1 *}$, Divya Vernekar² and Surabhi MY² \\ ${ }^{1}$ Lecturer in Speech Language Pathology, JSS Institute of Speech and Hearing, Dharwad, Karnataka, \\ India
}

${ }^{2}$ Students, III BASLP, JSS Institute of Speech and Hearing, Dharwad, Karnataka, India

Received: 08 June, 2020

Accepted: 20 June, 2020

Published: 22 June, 2020

*Corresponding author: Ms. Sandhya koteshwara, Lecturer in Speech Language Pathology, Department of Speech Language Pathology, JSS Institute of Speech and Hearing, Dharwad, Karnataka, India, Tel: 9035362883; E-mail: gangollibhoomi@gmail.com

ORCID: https://orcid.org/0000-0001-8329-0282

Keywords: Spasmodic dysphonia; Case report; Voice https://www.peertechz.com

Check for updates

\section{Abstract}

Spasmodic dysphonia is a neurogenic voice disorder which is characterized by severe and spasmodic problems of vocal fold approximation. Symptoms of the disorder consist of several sensations like strain and strangle which varies from mild to severe. Over the years, there have been several attempts in the line of treatment approaches which helps in voice modification by surgical, pharmacological treatment and voice therapy. In the recent years there are many success stories of patients who have benefitted from pharmacological treatments involving partial and temporary paralysis of muscle fibers within the larynx followed by botulinum injection. This article gives a picture of in depth evaluation of a 68 years old male who was diagnosed as having adductor spasmodic dysphonia. Detailed information regarding characteristics of voice, perceptual evaluation, acoustic analysis of voice, and medical and surgical management opted by the patient is discussed. Efficacy of voice therapy associated with medical management is explained in this case report. The case report emphasizes on the effectiveness of medical as well as therapeutic treatment required for spasmodic dysphonia as well as multifaceted approach to symptom management.

\section{Abbreviations}

SD: Spasmodic Dysphonia; RLN: Recurrent Laryngeal Nerve; NNE: Normalized Noise Energy; GRBAS: Grade, Roughness, Breathiness, Asthenic and Strain; CAPE-V: Consensus Auditory - Perceptual Evaluation - Voice; VHI: Voice Handicap Index

\section{Introduction}

Spasmodic dysphonia is a neurogenic voice disorder which is characterized by severe and spasmodic problems of vocal fold approximation [1]. In [2], first described spasmodic dysphonia as focal laryngeal dystonia. In this, involuntary spasms in the laryngeal muscles cause intermittent voice breaks [3]. Spasmodic dysphonia is called as a heterogeneous disorder with the subtypes of neurogenic, psychogenic and idiopathic causes that vary in clinical presentation and response to therapy. It implies that the two more common adductor and abductor form within the spasmodic dysphonia have different pathophysiological characteristics [4]. There are adductor, abductor and mixed types of spasmodic dysphonia. In adductor $\mathrm{SD}$, spasmodic hyperadductions of the vocal folds produce voice breaks with a choked, strained quality. Abductor SD is less common, with hyperabduction (uncontrolled opening) of the vocal folds prolonging voiceless consonants before vowels which results in severe breathy voice. Very rarely, adductor and abductor spasms occur in the same patient. Voice tremor is often present with SD [5]. There is no cure for spasmodic dysphonia but however, there are some medical and non medical management options. Medical intervention includes recurrent laryngeal nerve resection (RLN), Botox injection, and laser treatment whereas non medical intervention may include voice therapy. RLN section and Botox injection have achieved the greatest success than any other SD treatment [6]. Voice therapy that focussed on chewing and chanting techniques, biofeedback and techniques to lessen the stressful communication situation were recommended by [7].

\section{Need for the study}

The case study helps to understand the options available for detailed evaluation and management of a patient with Spasmodic Dysphonia proper documentation. Reporting such findings assists professionals to understand better about 
detailed assessment and management of such challenging cases.

\section{Case report}

A client aged 68 years male came to the department with the complaint of change in voice and difficulty while speaking from past 14 years. By profession the client is an advocate, speaker, attends debates of political issues and a trained Carnatic singer. The client experienced mild pain at the level of larynx and head. He had breathing difficulties while speaking. He also had the habit of consuming 10 packets of areca nut in a day since 10 years. The patient used to become aphonic after a prolonged usage and poor voice quality was observed after continuous speech, and facial grimaces were also observed.

\section{Method}

Stroboscopic evaluation of the client revealed glottic overclosure, presence of jerky movements, laryngeal tremors, hyperaduction in supraglotic stuctures and presence of adductor spasms.. Videolaryngoscopic report also revealed jerky movements of the vocal cords with the base of tongue and bilateral valleculae being normal. There was no obvious mass lesion. Acoustic data were gathered from voice samples of sustained phonation for about 5-8 seconds of the vowel /a/ at a comfortable pitch and loudness. The voice signals were recorded using a sensitive Panasonic boom microphone at a distance of 1 foot from the subject's mouth. The best sample out of the three with less background noise, better stability and good quality sample was considered for the further detailed analysis.

Perceptual voice evaluation was carried out by using GRBAS protocol [8], which assesses voice quality using four point rating scale ( Grade: equivalent to overall severity, Roughness, Breathiness, Asthenicity : weakness and Strain. In addition CAPE-V (Consensus Auditory - Perceptual Evaluation Voice) protocol which is a visual analog scale [9] was used to support perceptual evaluation which assesses Overall severity, roughness, breathiness, strain, pitch, loudness. Speech intelligibility was perceptually evaluated based on 7-point intelligibility rating scale. Objective voice assessment was done using Tiger Electronics' (Seattle, WA) Dr. Speech software (Voice Assessment, Version 3.0). The Fundamental frequency Fo $(\mathrm{Hz})$, mean Fo $(\mathrm{Hz})$, percent jitter, percent shimmer, and Normalized Noise Energy (NNE) were measured.

After detailed evaluation and appropriate diagnosis, the patient was recommended for regular voice therapy. Techniques such as relaxation, breathing exercises, resonant voice therapy, yawn and sigh and open mouth approach were oriented to the client and the patient was asked to follow during the therapy sessions and also while public speaking. Medical interventions such as recurrent laryngeal nerve resection (RLN section), Botox injection, and ML scopy-Laser Thyroarytenoid Myoneurectomy were also carried out.

The RLN section procedure involves surgical removal of a segment of one side of the RLN. The aim of this procedure is to reduce the glottis tightness in adductor spasmodic dysphonia which allows easy vocal production and intelligibility $[10,11]$, reported that the side effects of this procedure may include breathiness and aspiration. Botox injection for adductor spasmodic dysphonia results in substantial degree of improvement where the benefits lasts three to four months generally. Botox injection can also be an effective treatment for individuals who have had RLN section for treating adductor spasmodic dysphonia with the subsequent returns of signs and symptoms of the disorder. In thyroarytenoid myoneurectomy, with the help of $\mathrm{CO}_{2}$ laser the thyro arytenoid muscle fibers are destroyed. This shows significant long-term improvement in voice quality in terms of reduced speech breakes, effort and strain in voice.

\section{Results}

Perceptual evaluation results revealed a total score of 3 on Grade in GRBAS which was interpreted as severe breathy voice. The scores of CAPE V indicated moderately severe hoarse voice. Speech Intelligibility was rated as 6 which is interpreted as speech occasionally understood. Dr.speech values showed significant increase in jitter shimmer and NNE which indicates severe voice abnormality. The diagnosis was made by correlating perceptual and objective evaluation results with medical findings which yielded the diagnosis of severe hoarse voice secondary to adductor spasmodic dysphonia.

The patient attended voice therapy for 25 sessions. Marked improvement was noticed in voice therapy with the medical treatment. Objective evaluation results showed decreased jitter and shimmer and NNE values which indicates reduced breathy component in voice (Graph 1). Breathy, strain and asthenic components of GRBAS were reduced (Graph 2). A post therapy perceptual voice rating revealed a score of 1 indicating mild hoarse voice. CAPE V scores indicated mild hoarse voice.

\section{Discussion}

With regular voice therapy along with the medical line of treatment showed a significant improvement in voice. This study demonstrates that patients with spasmodic dysphonia significantly improve in their acoustic and perceptual characteristics after medical and non medical intervention. In support of the current study, a research by [12], a combinedmodality treatment program containing botulinum toxin injection (Botox) and voice therapy was used to treat 17

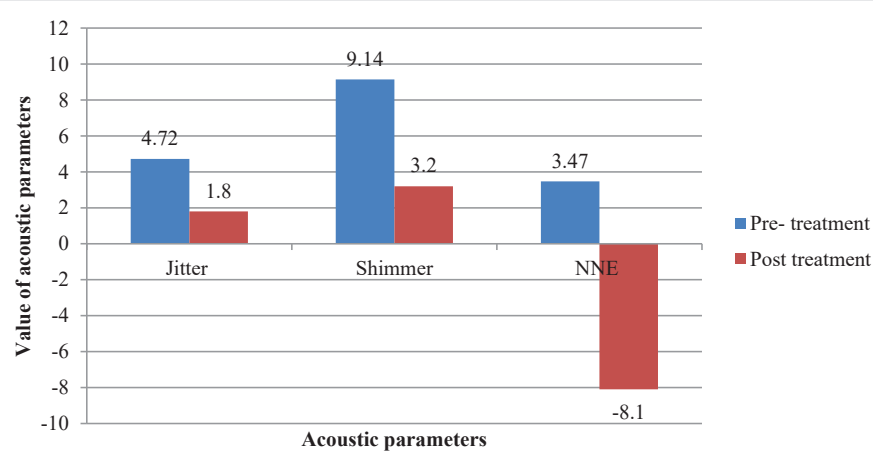

Figure 1: Comparison of Fundamental frequency (F0), Jitter, Shimmer and NNE: Pre and post treatment.

Citation: Sandhya K, Divya V, Surabhi MY (2020) Adductor spasmodic dysphonia - A detailed case report on assessment and management. Glob J Medical Clin Case Rep 7(1): 051-053. DOI: https://dx.doi.org/10.17352/2455-5282.000098 


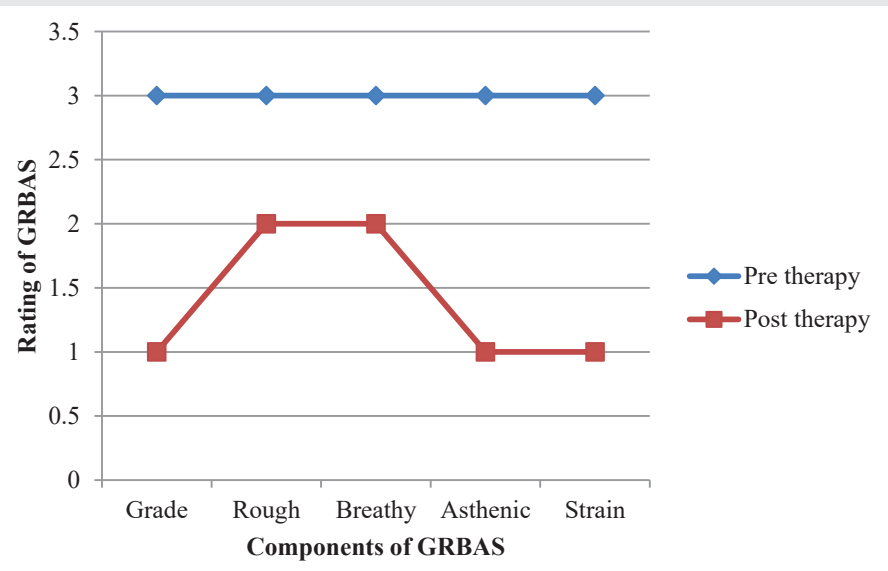

Figure 2: Comparison of GRBAS pre and post treatment scores.

subjects diagnosed with adductor spasmodic dysphonia (ADD $\mathrm{SD})$. Voice therapy after Botox injection was directed to reduce the hyperfunctional vocal behaviors, glottal overpressure at voice onset and anterior-posterior squeezing. The results indicated that subjects who underwent combined-modality treatment showed significantly higher mean airflow rates for significantly longer periods. In another study by [13], Botulinum toxin injections were used to treat 31 patients with adductor spasmodic dysphonia. This treatment showed significant reduction in the standard deviation of the fundamental frequency of the speech sample and also indicated a reduction in the variability of pitch amongst patients. A total of $96 \%$ of patients subjective diary reports showed an improvement with a median of 7 days to peak effect, that is, the length of time the voice was at its maximum improvement before any deterioration was noted, and a 5 week duration of peak effect. According to the study by [14], with restricted role of speech therapy, several surgical modalities have been used with a huge success rate. Objective of the study was to document the outcome of voice quality followed by thyroarytenoidectomy in patients with adductor spasmodic dysphonia. The combined modality treatment program consisting of botulinum toxin injection (Botox), RLN resection, laser treatment along with voice therapy reduced the intrinsic laryngeal muscle spasms and improved the hyper functional vocal behaviors. Although it is difficult to find a permanent cure for Spasmodic Dysphonia, it is possible to show betterment in voice and decrement in regular spasms by using appropriate medical intervention combined with regular and effective voice therapy techniques.

\section{Consent}

Written consent was taken from the patient to undergo voice evaluation and to be involved in research.

\section{References}

1. Szkielkowska A, Miaskiewicz B, Baczynska-Swierczynska S, Szymanska E (2006) Therapeutic Difficulties in Spasmodic Dysphonia--Case Report. Pol Merkur Lekarski 20: 557-559. Link: https://bit.ly/3emt1AQ

2. Traube L (1871) Spastische form der nervoesen heiserkeit. Gesammelte beitraege zur pathologies und physiologie. Vol. 2. Berlin: Hirschwald.

3. Shipp T, Izdebski K, Reed C, Morrissey P (1985) Intrinsic laryngeal muscle activity in a spastic dysphonic patient. J Speech Hear Disord 50: 54-59. Link: https://bit.ly/3dpWF7j

4. Aronson AE (1985) Clinical Voice Disorders ( $2^{\text {nd }}$ ed.). Thieme, NewYork

5. Edgar JD, Sapienza CM, Bidus K, Ludlow CL (2001) Acoustic measures of symptoms in abductor spasmodic dysphonia. J Voice 15: 362-372. Link: https://bit.ly/3dleOD3

6. Miller RH, Woodson GE (1991) Treatment options in spasmodic dysphonia. Otolaryngologic Clinics of North America 24: 1227-1236. Link: https://bit.ly/2ATc8PR

7. Prater RJ, Swift RW (1984) Manual of voice therapy. Austin TX.

8. Hirano M (1981) Clinical examination of the voice. New York, NY: SpringerVerlag. Link: https://bit.ly/2Bt8BHK

9. Kempster GB, Gerratt BR, Verdolini Abbott K, Barkmeier-Kraemer J, Hillman RE (2009) Consensus auditory-perceptual evaluation of voice: Development of standardized clinical protocol. American Journal of Speech Language Pathology 18: 124-132. Link: https://bit.ly/2zLHMy6

10. Hugh Milton Miller (1955) Forty Wayes of 2 Pts. in One of Thomas Woodson. Journal of the American Musicological Society.

11. Zwirner P, Murray T, Swenson M, Woodson GE (1992) Effects of botulinum toxin therapy in patients with adductor spasmodic dysphonia: Acoustic, aerodynamic and video endoscopic findings. Laryngoscope 102: 400-406. Link: https://bit.ly/2YSqbNt

12. Zwirner P, Murray T, Swenson M, Woodson GE (1991) Acoustic changes in spasmodic dysphonia after botulinum toxin injection. J Voice 5: 78-84. Link: https://bit.ly/30XREA8

13. Whurr R, Lorch M, Fontana H, Brookes G, Lees A, et al. (1993) The use of botulinum toxin in the treatment of adductor spasmodic dysphonia. J Neurol Neurosurg Psychiatry 56: 526-530. Link: https://bit.ly/2V26iCN

14. Gandhi S, Remacle M, Mishra P, Desai V (2014) Vocal outcome after endoscopic thyroarytenoid myoneurectomy in patients with adductor spasmodic dysphonia. Eur Arch Otorhinolaryngol 271: 3249-3254. Link: https://bit.ly/37IPS7p

Copyright: @ 2020 Sandhya K, et al. This is an open-access article distributed under the terms of the Creative Commons Attribution License, which permits unrestricted use, distribution, and reproduction in any medium, provided the original author and source are credited. 